\title{
Twister: Construction and structural relaxation of commensurate moiré superlattices
}

\author{
Saismit Naik ${ }^{\mathrm{a}}$, Mit H. Naik ${ }^{\mathrm{b}}$, Indrajit Maity ${ }^{\mathrm{b}}$, Manish Jain ${ }^{\mathrm{b}, *}$ \\ ${ }^{a}$ Indian Institute of Science Education and Research, Pune 411008, India \\ ${ }^{b}$ Centre for Condensed Matter Theory, Department of Physics, Indian Institute of \\ Science, Bangalore 560012, India
}

\begin{abstract}
Introduction of a twist between layers of two-dimensional materials which leads to the formation of a moiré pattern is an emerging pathway to tune the electronic, vibrational and optical properties. The fascinating properties of these systems is often linked to large-scale structural reconstruction of the moiré pattern. Hence, an essential first step in the theoretical study of these systems is the construction and structural relaxation of the atoms in the moiré superlattice. We present the Twister package, a collection of tools that constructs commensurate superlattices for any combination of $2 \mathrm{D}$ materials and also helps perform structural relaxations of the moiré superlattice. Twister constructs commensurate moiré superlattices using the coincidence lattice method and provides an interface to perform structural relaxations using classical forcefields.
\end{abstract}

Keywords: Coincidence Lattice Theory, Commensurate superlattice, moiré patterns, 2D materials

\footnotetext{
PROGRAM SUMMARY

Program Title: Twister

Program obtainable from: https://github.com/qtm-iisc/Twister

Journal Reference:

Catalogue identifier:

Licensing provisions: Open source BSD License
}

\footnotetext{
*Corresponding author.

E-mail address: mjain@iisc.ac.in
} 
Programming language: Python

Computer: Any computer with Python3 installed. The package has been tested with Python3.6

Operating system: Unix/Linux/Windows

RAM: 5-1000 MB (dependent on system size)

Keywords: Coincidence Lattice Theory, Commensurate superlattice, heterobilayer 2D material, Strain corrections, Electronic Structure

Classification:

External routines/libraries: numpy, scipy, mpi4py, matplotlib

Nature of problem:

Solution method:

Running time: 1-600 minutes (depends on the number of processors and system size)

\section{Introduction}

Two-dimensional (2D) materials, with their extensive spectrum of physical properties and long reaching applicability in technology, have triggered a wave of innovation in the realm of material science. Twisting the layers of $2 \mathrm{D}$ materials to form moiré patterns is an emerging direction of research 1, 2, 3, 4, 5, 6, 7, 8, 9, 10, which was triggered by the recent discovery of unconventional superconductivity and Mott-insulating phases in twisted bilayer graphene [11, 12. An important feature of the moiré patterns is that they undergo structural reconstructions as predicted by theoretical simulations [13, 14, 15, 16, 17, 18, 19, 20, 21, 22, 23, 24, 25] and corroborated by experiments [26, 27, 28, 29, 30, 31, 32. The atomic reconstructions can strongly modify the electronic structure [28, 16, 33, 30, 29, 34, 35, 17, 36, 37, 38, 39] and vibrational modes [40, 41, 42] of the moiré superlattice.

Most theoretical methods, particularly those based on classical forcefields or first-principles density functional theory [43] (DFT), require the creation of a periodic simulation cell. Application of an arbitrary twist between the layers of a 2D material leads to an incommensurate moiré which is intractable for these methods. It is thus essential to find special twist angles [44, 45] which lead to moiré superlattices that are commensurate with periodic boundary conditions. A common approach to find these twist angles is the coincidence lattice method [44, 46]. In this method, the commensurate twist angle is determined as the one at which perfect coincidence occurs for a set of lattice 
points in the top and bottom layers. This method has been used to derive an analytical expression for the commensurate twist-angles in systems which have a hexagonal lattice and exactly same lattice constant in the top and bottom layer. To go beyond this restricted class to $2 \mathrm{D}$ materials, a general implementation of this method is necessary. Furthermore, it is important to note that perfect coincidence of lattice sites is often not possible or only possible at very large length scales. In this case a small strain may be applied to each layer to obtain a commensurate superlattice of reasonable size [46].

At large moiré length scales, all moiré superlattices undergo structural reconstructions arising from in-plane and out-of-plane displacement of atoms from the rigidly-twisted structure. In-plane displacements lead to a change in the local stackings giving rise to strain-soliton networks [17, 16, 13, 14, 26, 30] and out-of-plane displacements lead to a varying interlayer spacing and/or buckling of the layers [29, 47, 48, 49, 50] in the moiré. Hence, once the commensurate moiré superlattice is constructed, performing structural relaxation is the essential next step before exploring its electronic or optical properties. Despite evidence of structural reconstructions, many theoretical studies of moiré superlattices do not take this into account [10, 51, 52, 53, 54, 55]. This is because lattice relaxations using van der Waals corrected DFT is a major computational bottleneck due to the large number of atoms that constitute the moiré superlattice. An alternative computationally cheaper approach to perform the structural relaxation of the moire superlattices is based on classical forcefields. This approach eliminates the electronic degrees of freedom and employs a theoretical description involving only nuclei. The interactions between nuclei are represented with simple inter-atomic potentials, whose parameters are obtained by fitting to accurate DFT calculations. For instance, the structural relaxations of the moiré superlattices of twisted bilayer of transition metal dichalcogenides (TMDs) are performed with a combination of Stillinger-Weber [56] (SW) and registry-dependent KolmogorovCrespi [57] (KC) potentials. The SW and KC potential describes intralayer and interlayer interaction in these systems. The SW potential parameters were obtained by fitting to DFT-derived phonon dispersion of single layer TMD [58, whereas the KC potential parameters were obtained by fitting interlayer binding energy landscape to van der Waals corrected DFT results [59, 60, 61, 62, 63]. The fit forcefields can be used to obtain moiré reconstructions in good agreement with DFT relaxations [59] at a fraction of the computational cost. However, the technical details of classical force-field based simulation are non-trivial. An all-inclusive package that constructs 
and automates the performance of lattice relaxations of the moiré superlattice can bridge this gap for non-experts.

We present the Twister package to construct commensurate moiré superlattices for any 2D homobilayer or heterobilayer material. Twister helps find minimum-area commensurate superlattices using the coincidence lattice method for a given range of twist angles and user-defined strain tolerance. The superlattice vectors and atoms within the superlattice are written to a file for further study of its structural and electronic properties. The construction of commensurate superlattice has been tested for various homobilayer and heterobilayer cases: graphene/hBN, $\mathrm{MoS}_{2} / \mathrm{MoSe}_{2}, \mathrm{hBN} / \mathrm{MoSe}_{2}$, twisted bilayer phosphorene and $\mathrm{MoS}_{2}$. We use the package to find a range of commensurate twist angles for the case of $\mathrm{MoS}_{2} / \mathrm{MoSe}_{2}$ and twisted bilayer phosphorene. Twister further also streamlines the process of studying moiré reconstruction using classical forcefield calculations as implemented in the LAMMPS [64] package. The streamlined process is demonstrated for twisted bilayer $\mathrm{MoS}_{2}$, twisted bilayer graphene and twisted $\mathrm{MoS}_{2} / \mathrm{MoSe}_{2}$ moiré patterns.

\section{Construction of commensurate superlattices}

The method used to construct the commensurate superlattices depends on the type of lattice vectors in the bottom and top layer forming the moiré. If the two lattices are hexagonal and with identical lattice constants, we use an analytical expression [45] to obtain the commensurate twist-angles $\left(\theta_{i}\right)$.

$$
\cos \left(\theta_{i}\right)=\frac{3 i^{2}+3 i+0.5}{3 i^{2}+3 i+1}
$$

where $i$ is a whole number. The corresponding superlattice vectors are given by: $\mathbf{A}_{1}=i \mathbf{a}_{1}+(i+1) \mathbf{a}_{2}, \mathbf{A}_{2}=-(i+1) \mathbf{a}_{1}+(2 i+1) \mathbf{a}_{2}$ where $\mathbf{a}_{1}$ and $\mathbf{a}_{2}$ are the unit-cell lattice vectors. In the superlattices generated using this equation, the peridocity of the stackings matches the moiré unit-cell periodicity. Ie. each type of stacking (say AA) is present once per moiré unit-cell. For commensurate twist-angles in addition to $\theta_{i}$, a general form for commensurate twist-angles is given by [44]:

$$
\cos \left(\theta_{m, n}\right)=\frac{n^{2}+4 n m+m^{2}}{2\left(n^{2}+m n+m^{2}\right)},
$$


where $\mathrm{m}, \mathrm{n}$ are whole numbers. The superlattice vectors are: $\mathbf{A}_{1}=m \mathbf{a}_{1}+$ $n \mathbf{a}_{2}, \mathbf{A}_{2}=-n \mathbf{a}_{1}+(m+n) \mathbf{a}_{2}$. This general equation yields all possible commensurate superlattices and not just ones with minimum area. The superlattice can have mutiple AA stackings per moiré unit-cell.

For a combination of layers with dissimilar lattices we use the coincidence site lattice theory to generate the superlattice. Consider the unit-cell lattice vectors of bottom layer to be $\mathbf{a}_{1}, \mathbf{a}_{2}, \mathbf{a}_{3}$ and of top layer to be $\mathbf{b}_{1}, \mathbf{b}_{2}, \mathbf{b}_{3}$. Let $\mathbf{R}_{\theta}$ be the rotation matrix corresponding to a rotation angle $\theta$ in the xy plane. Top layer is rotated about the origin keeping the bottom layer fixed. The rotated lattice vectors, $\mathbf{b}_{i}^{r}$ are given by:

$$
\mathbf{b}_{i}^{r}=\mathbf{b}_{\mathbf{i}} \mathbf{R}_{\theta}^{\mathbf{T}}
$$

Coincidence of a subset of lattice sites occurs when a lattice vector in bottom layer, $\mathbf{v}_{1}=n_{1} \mathbf{a}_{1}+n_{2} \mathbf{a}_{2}$, superposes with a lattice vector in the rotated top layer, $\mathbf{v}_{2}^{r}=m_{1} \mathbf{b}_{1}^{r}+m_{2} \mathbf{b}_{2}^{r}$, ie.

$$
n_{1} \mathbf{a}_{1}+n_{2} \mathbf{a}_{2}=m_{1} \mathbf{b}_{1}^{r}+m_{2} \mathbf{b}_{2}^{r}
$$

Figure 1 graphically represents the above steps for $27.8^{\circ}$ twisted bilayer $\mathrm{MoS}_{2}$. The shortest coinciding vectors have been plotted and any two non-parallel vectors can be equivalently used as superlattice vectors for the given twist angle.

For heterobilayer materials, it is often difficult to obtain exact coincidence since lattice parameters of the constituent layers are different. Hence, we introduce a user-defined upper limit of mismatch, $\Delta$, between the vectors in $\AA$ units,

$$
\left|\mathbf{v}_{1}-\mathbf{v}_{2}^{r}\right| \leq \Delta
$$

It is important to find superlattices with as small a mismatch as possible to ensure that the superlattice is commensurate with the twist-angle. A large value of $\Delta$ can introduce spurious strains in the superlattice. For an arbitrary combination of layers, the value of mismatch or the size of superlattice is often too large despite sweeping a large range of twist-angles. In these cases, more effort is needed to find perfectly commensurate superlattices. In the following subsections, we describe two methods to improve coincidence.

After the computation of all vectors co-incident within the mismatch, pairs of solutions ( $\left.\left\{n_{1} \mathbf{a}_{1}+n_{2} \mathbf{a}_{2}, n_{1}^{\prime} \mathbf{a}_{1}+n_{2}^{\prime} \mathbf{a}_{2}\right\}\right)$ are checked to be non-parallel by ensuring their vector cross products is non-zero. The solutions can also 
(a)

- Bottom Layer

- Top Layer

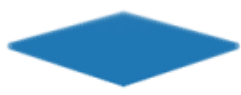

(b)

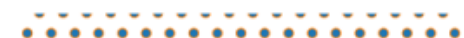

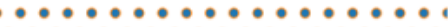

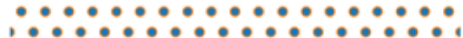

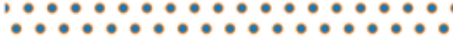

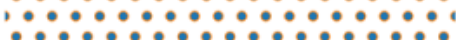

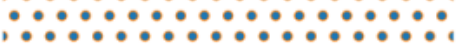

$\because \therefore \because \therefore \because \therefore \because \therefore \because \therefore \because$

$\because \because \because \because \because \cdots \cdots \cdots \cdots$

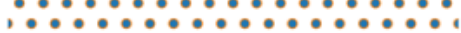

$\because \therefore \because \therefore \because \therefore \because \therefore \because$

$\because \because \therefore \because \because \because \because \because \because \because \because \because$

$\because \because \because \because \because \because \because \because \because \because \cdots \cdots$

$\because \because \therefore \because \therefore \because \therefore \because \therefore \because \because 8$

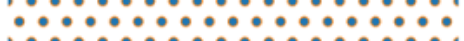

$\because \therefore \therefore \therefore \therefore \therefore \therefore \because \therefore \because \therefore \because \because \because 9$ (c)

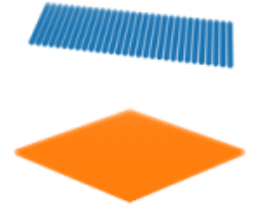

(d)

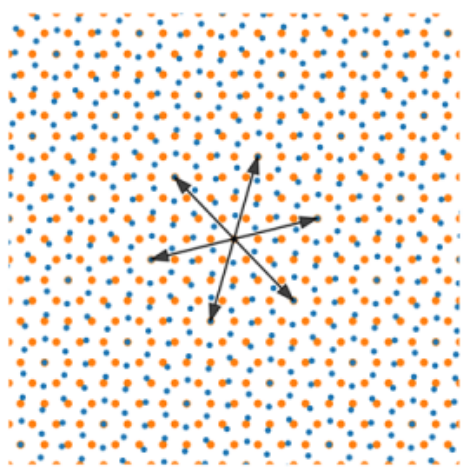

Figure 1: a) and c) 3-D representations of untwisted and twisted $\mathrm{MoS}_{2}$ homobilayer respectively. Twist angle is $27.8^{\circ}$ in c). b) and d) Top view of a) and c), respectively. Six solution vectors of Eqn. 4 (with minimum superlattice area) are shown in d)

be further restricted to have a specific angle between the vectors. In the final step, we choose superlattices with minimum area with a variation of $1 \%$ allowed around the minima. The final output is a list of appropriate superlattice vectors and the corresponding strained lattice parameters.

\subsection{Straining lattice parameters}

The lattice parameters of each layer can be uniformly strained as demonstrated for the case of $\mathrm{hBN} / \mathrm{MoSe}_{2}$ in Fig. 2. A biaxial strain $(\beta)$ is applied to each layer such that the following equation is satisfied [46]:

$$
(1+\beta)\left|\mathbf{v}_{1}\right| \approx(1-\beta)\left|\mathbf{v}_{2}^{r}\right|
$$

Lattice parameters of one layer are strained by $\beta$ and those of the lower layer by $-\beta$ to improve coincidence. In certain situations, it is desirable to 
allow for only one layer to be strained. If only lattice parameters of the top layer are to be strained, $\beta$ is applied to the top layer such that:

$$
\left|\mathbf{v}_{1}\right| \approx(1+\beta)\left|\mathbf{v}_{2}^{r}\right|
$$

If only lattice parameters of the bottom layer are to be strained, $\beta$ is applied to the bottom layer such that:

$$
(1+\beta)\left|\mathbf{v}_{1}\right| \approx\left|\mathbf{v}_{2}^{r}\right|
$$

Two values of $\beta$ are generated for each pair of solutions $\left(\left\{n_{1} \mathbf{a}_{1}+n_{2} \mathbf{a}_{2}, n_{1}^{\prime} \mathbf{a}_{1}+\right.\right.$ $\left.\left.n_{2}^{\prime} \mathbf{a}_{2}\right\}\right)$ and the averaged value is used to form the superlattice vectors. The mismatch after straining the superlattice vectors is computed as demonstrated in Eqn. 5 .

\subsection{Applying a strain tensor}

When straining the lattice parameters alone does not yield small mismatch, the unit-vectors of the top layer can be deformed to ensure perfect coincidence. However, this may change the angle between the lattice vectors of the strained layer leading to breaking of symmetries within that layer. The deformation tensor $(\boldsymbol{D})$ is computed such that:

$$
\left[\begin{array}{ll}
n_{1} & n_{2} \\
n_{1}^{\prime} & n_{2}^{\prime}
\end{array}\right]\left[\begin{array}{l}
\mathbf{a}_{1} \\
\mathbf{a}_{2}
\end{array}\right]=\left[\begin{array}{ll}
m_{1} & m_{2} \\
m_{1}^{\prime} & m_{2}^{\prime}
\end{array}\right]\left[\begin{array}{l}
\mathbf{b}_{1} \\
\mathbf{b}_{2}
\end{array}\right] \boldsymbol{D} \boldsymbol{R}_{\boldsymbol{\theta}}^{\boldsymbol{T}}
$$

Any deformation of a lattice can be expressed in terms of a pure rotation of the lattice and a symmetric strain tensor. Through a polar decomposition 65], we have $\boldsymbol{D}=\boldsymbol{\epsilon} \boldsymbol{R}_{\phi}$ :

$$
\left[\begin{array}{ll}
n_{1} & n_{2} \\
n_{1}^{\prime} & n_{2}^{\prime}
\end{array}\right]\left[\begin{array}{l}
\mathbf{a}_{1} \\
\mathbf{a}_{2}
\end{array}\right]=\left[\begin{array}{ll}
m_{1} & m_{2} \\
m_{1}^{\prime} & m_{2}^{\prime}
\end{array}\right]\left[\begin{array}{l}
\mathbf{b}_{1} \\
\mathbf{b}_{2}
\end{array}\right] \boldsymbol{\epsilon} \boldsymbol{R}_{\phi} \boldsymbol{R}_{\boldsymbol{\theta}}^{\boldsymbol{T}}
$$

The pure rotation leads to a further change in the twist-angle by $\phi$. The change in the twist-angle along with a strain in the unit-vectors (by $\boldsymbol{\epsilon}$ ) leads to a perfectly commensurate superlattice.

\section{Test Systems}

The construction of commensurate superlattices using our implementation (Eqn 4) is verified for five test systems - twisted bilayer $\mathrm{MoS}_{2}, \mathrm{MoS}_{2} / \mathrm{MoSe}_{2}$ heterobilayer, Graphene/hBN heterobilayer and hBN/MoSe ${ }_{2}$ heterobilayer 


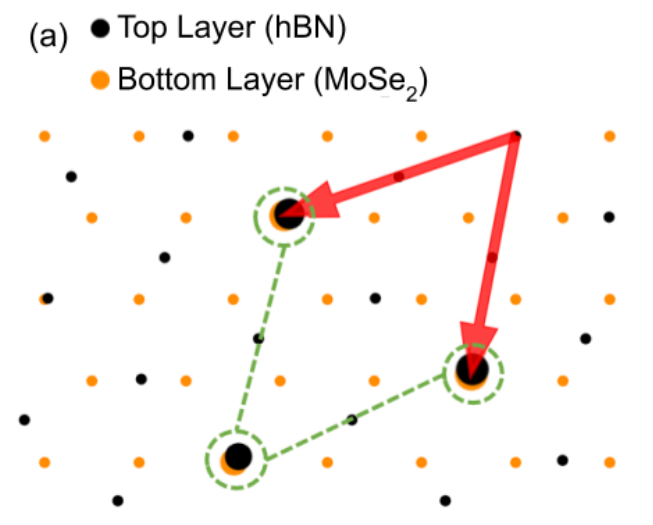

(b)

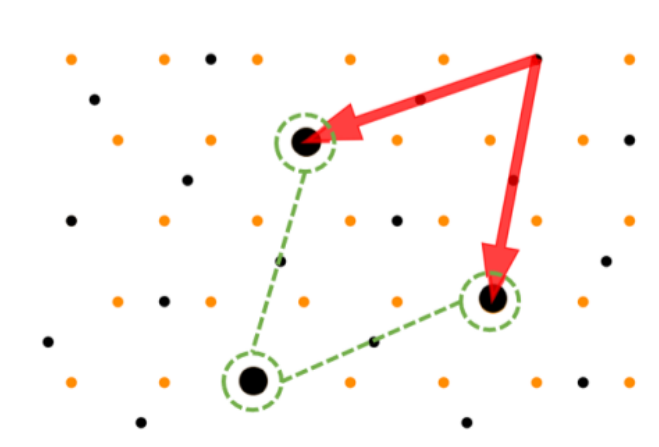

Figure 2: Red arrows are the superlattice vectors of $\mathrm{hBN} / \mathrm{MoSe}_{2}$ bilayer twisted at $19.1^{\circ}$. The corner lattice points are encircled. a) Supercell with unstrained lattice parameters. b) Supercell with strained parameters with improved overlap.

to compare with Ref. [46]. To find the commensurate superlattices (using the procedure described in section 2) we use the following constraints. For $\mathrm{MoS}_{2} / \mathrm{MoSe}_{2}, \mathrm{MoS}_{2} / \mathrm{MoS}_{2}$ and hBN/MoSe 2 , the angle between superlattice vectors is fixed at $60^{\circ}$. For Graphene/hBN, the angle between superlattice vectors is fixed to be $120^{\circ}$. Maximum strain, $\beta$, allowed in the lattice parameters is $1 \%$. Mismatch $(\AA)$ threshold, $\Delta$, is set to be less that $0.1 \AA$. The commensurate superlattices, number of atoms and strains are in agreement with the reported results [46, 33]. The lattice parameters used in our simulation and the results are shown in Table 1 and Table 2 , respectively. The input files along with instructions on how to use the code to generate the commensurate superlattices for these test systems is included in the Twister package.

\section{Examples}

\subsection{Commensurate twist-angles}

Apart from the above test examples, we use Twister to compute commensurate twist-angles for the heterobilayer systems of $\mathrm{MoS}_{2} / \mathrm{MoSe}_{2}$ and 


\begin{tabular}{cr}
\hline \hline Materials & Lattice parameter $(\AA)$ \\
\hline $\mathrm{MoS}_{2}$ & 3.164 \\
$\mathrm{MoSe}_{2}$ & 3.301 \\
Graphene & 2.467 \\
hBN & 2.512 \\
Phosphorene & $3.321,4.62$ \\
\hline
\end{tabular}

Table 1: Lattice parameters of each material

\begin{tabular}{crrrr}
\hline \hline Materials & Twist angle Superlattice vectors Atoms Biaxial strain \\
& \multicolumn{4}{c}{$\left(n_{1}, n_{2}\right),\left(n_{1}^{\prime}, n_{2}^{\prime}\right)$} \\
\hline $\mathrm{MoS}_{2} / \mathrm{MoS}_{2}$ & $27.8^{\circ}$ & $(-4,3),(-3,-1)$ & 78 & \pm 0.0 \\
$\mathrm{MoS}_{2} / \mathrm{MoSe}_{2}$ & $16.1^{\circ}$ & $(-1,-3),(3,-4)$ & 75 & \pm 0.119 \\
$\mathrm{Graphene}_{\mathrm{hBN}}$ & $0.0^{\circ}$ & $(-37,0),(0,37)$ & 5330 & \pm 0.466 \\
$\mathrm{hBN} / \mathrm{MoSe}_{2}$ & $19.1^{\circ}$ & $(1,-3),(3,-2)$ & 26 & \pm 0.333 \\
\hline
\end{tabular}

Table 2: Results obtained from Twister for the test systems to compare with the commensurate twist-angles reported in Ref. [46].

bilayer phosphorene. For systems of $\mathrm{MoS}_{2} / \mathrm{MoSe}_{2}$, the angle between superlattice vectors is fixed at $60^{\circ}$. Strain for lattice parameters is kept below $1 \%$ and distributed over both layers. Mismatch threshold is $10^{-5} \AA$. The size of the moiré superlattice is expected to increase as a function of reducing twist-angle. We accordingly increase the search range of $n_{1}, n_{2}, m_{1}, m_{2}$ (Eqn. (4) as a function of decreasing twist-angle. The commensurate twist-angles for $\mathrm{MoS}_{2} / \mathrm{MoSe}_{2}$ can be found in Table 3. The commensurate twist-angles for twisted bilayer phosphorene are generated using the deformation tensor method described above. One of the layers is allowed to deform to form a perfectly commensurate superlattice. A fine tuning of the twist-angles is not necessary for this method. For example, starting with $\theta=6.9^{\circ}$ leads to the following strain and rotation matrices (Eqn. 10):

$$
\boldsymbol{\epsilon}=\left[\begin{array}{cc}
0.992893 & -0.001897 \\
-0.001897 & 0.993364
\end{array}\right] ; \boldsymbol{R}_{\phi}=\left[\begin{array}{cc}
0.999995 & -0.003102 \\
0.003102 & 0.999995
\end{array}\right]
$$

The $\boldsymbol{\epsilon}$ tensor is applied to the lattice vectors and the $\boldsymbol{R}_{\phi}$ leads to a change in the twist-angle between the layers by $\phi=-0.17773^{\circ}$. The commensurate twist-angle is thus, $\theta+\phi=6.72227^{\circ}$. To test the implementation, we also 
start with $\theta=6.5^{\circ}$ and find a solution:

$$
\boldsymbol{\epsilon}=\left[\begin{array}{cc}
0.992919 & -0.001900 \\
-0.001900 & 0.993338
\end{array}\right] ; \boldsymbol{R}_{\phi}=\left[\begin{array}{cc}
0.999992 & 0.003879 \\
-0.003879 & 0.999992
\end{array}\right]
$$

The strain tensor is nearly the same, while the change in twist-angle, $\phi=$ $0.2222699^{\circ}$. As expected, the final commensurate twist-angle $(\theta+\phi)$ remains identical for different starting values of $\theta$. The commensurate twist-angles for twisted bilayer phosphorene generated using this method are provided in Table 4. The input files, along with instructions on how to run the code, are provided with the Twister package. More details on using the code and the workflow is described in the next section.

\begin{tabular}{crrrr}
\hline \hline Twist Angle & Atoms & $\begin{array}{c}\text { Superlattice vectors } \\
(\mathrm{n} 1, \mathrm{n} 2),\left(\mathrm{n} 1^{\prime}, \mathrm{n} 2^{\prime}\right)\end{array}$ \\
\hline $0.0^{\circ}$ & 3315 & $(24,0),(0,24)$ & 0.00856 & $10^{-9}$ \\
$1.56654^{\circ}$ & 2283 & $(11,-23),(-12,-11)$ & 0.0501 & $5 \times 10^{-6}$ \\
$2.84558^{\circ}$ & 1362 & $(4,-17),(17,-13)$ & 0.0847 & $2 \times 10^{-6}$ \\
$3.10565^{\circ}$ & 1248 & $(-16,13),(-13,-3)$ & 0.0454 & $2 \times 10^{-6}$ \\
$4.715^{\circ}$ & 696 & $(0,-11),(11,-11)$ & 0.0371 & $2 \times 10^{-6}$ \\
$7.3111^{\circ}$ & 327 & $(-8,1),(-7,8)$ & 0.176 & $4 \times 10^{-6}$ \\
$11.17856^{\circ}$ & 456 & $(3,-10),(10,-7)$ & 0.145 & $1 \times 10^{-6}$ \\
$14.11315^{\circ}$ & 384 & $(7,-9),(-2,-7)$ & 0.226 & $2 \times 10^{-6}$ \\
$16.1021^{\circ}$ & 75 & $(-4,1),(-3,4)$ & 0.118 & $3 \times 10^{-6}$ \\
$21.45415^{\circ}$ & 384 & $(7,-9),(-2,-7)$ & 0.226 & $10^{-6}$ \\
\hline
\end{tabular}

Table 3: Commensurate twist-angles in the range $0-30^{\circ}$ generated for $\mathrm{MoS}_{2} / \mathrm{MoSe}_{2}$ using Twister. Lattice parameters were strained to improve mismatch.

\subsection{Structural relaxations}

We demonstrate the automation of construction and relaxation of moiré superlattices (using the LAMMPS package) for the case of twisted bilayer $\mathrm{MoS}_{2}$. Using a single script, homo.sh, a range of commensurate superlattices can be generated and relaxed. Using this script we perform the following:

1. A file, hex.table, which contains a series of twist angles and superlattice vectors is created. 


\begin{tabular}{|c|c|c|c|c|c|}
\hline Twist Angle & Atoms & $\begin{array}{l}\text { erlattice vectors } \\
1, \mathrm{n} 2),\left(\mathrm{n} 1{ }^{\prime}, \mathrm{n} 2\right. \\
\end{array}$ & & Strain tensor & Mismatch $(\AA)$ \\
\hline $1.595323^{\circ}$ & 10444 & $(45,0),(0,29)$ & $\begin{array}{l}1.00047 \\
0.00306\end{array}$ & $\left.\begin{array}{ll}7 & 0.00306 \\
6 & 1.00030\end{array}\right]$ & 0.0 \\
\hline $2.760976^{\circ}$ & 3460 & $(27,0),(0,16)$ & $\begin{array}{l}0.99901 \\
0.0032 £\end{array}$ & $\begin{array}{ll}1 & 0.00329 \\
8 & 0.99869\end{array}$ & 0.0 \\
\hline $3.523156^{\circ}$ & 2116 & $(22,0),(0,12)$ & $\begin{array}{l}0.99821 \\
0.00166\end{array}$ & $\begin{array}{ll}1 & 0.00166 \\
6 & 0.99801\end{array}$ & 0.0 \\
\hline $4.265478^{\circ}$ & 1444 & $(18,0),(0,10)$ & $\begin{array}{l}0.99744 \\
0.00268\end{array}$ & $\begin{array}{ll}4 & 0.00268 \\
8 & 0.99704\end{array}$ & 0.0 \\
\hline $5.404710^{\circ}$ & 900 & $(14,0),(0,8)$ & $\begin{array}{l}0.99602 \\
0.0046 ؛\end{array}$ & $\begin{array}{ll}2 & 0.00469 \\
9 & 0.99513\end{array}$ & 0.0 \\
\hline $6.722269^{\circ}$ & 580 & $(12,0),(0,6)$ & $\begin{array}{c}0.99289 \\
-0.00189\end{array}$ & $\begin{array}{c}-0.00189 \\
0.99337\end{array}$ & 0.0 \\
\hline
\end{tabular}

Table 4: Commensurate twist-angles of bilayer black phosphorous generated using Twister. A strain tensor is applied to one of the layers to improve mismatch.

2. The moiré superlattice and atom positions are generated for each twistangle in this file.

3. To perform the relaxation using LAMMPS, the superlattice vectors must be reoriented so that one of the superlattice vectors is along the (100) direction. Consider three superlattice vectors of the moiré superlattice generated using Eqn. 1; $\mathbf{v}_{1}, \mathbf{v}_{2}$ and $\mathbf{v}_{3}$. $\hat{\mathbf{v}}_{3}$ is along the out-of-plane, $z$ direction. The transformation is thus computed in the following manner,

$$
\begin{gathered}
\vec{A}_{1}=\left\|\vec{v}_{1}\right\| \hat{x} \\
\vec{A}_{2}=\left(\vec{v}_{2} \cdot \hat{v}_{1}\right) \hat{x}+\left(\left|\hat{v}_{1} \times \vec{v}_{2}\right|\right) \hat{y} \\
\vec{A}_{3}=\vec{v}_{3}
\end{gathered}
$$


4. With the user-provided force-fields, the atomic relaxations are performed using the LAMMPS package for all the twist-angles in hex.table.

The range of twist-angles in hex.table can be generated using both Eqn. 1 or 2 according to the user's preference. The relaxed structures can be visualized with the OVITO [66] software directly. The order-parameter and interlayer spacing distribution for relaxed $1.61^{\circ}$ and $58.39^{\circ}$ twisted bilayer $\mathrm{MoS}_{2}$ is shown in Fig. 3. The order-parameter is defined as the shortest displacement of the top layer that transforms a given local stacking to the highest energy stacking in the moiré - AA for $1.61^{\circ}$ twist and $\mathrm{A}^{\prime} \mathrm{B}$ for $58.39^{\circ}$ twist. The relaxation leads to increase in the area of low-energy stackings (AB, BA, AA', AB') and formation of shear-strain solitons at the boundary between adjacent low-energy stackings [1, 17, 36, 13. The strain distribution in the bottom layer of the relaxed moiré pattern is shown in Fig. 4. The strains are localised along the solitonic networks.
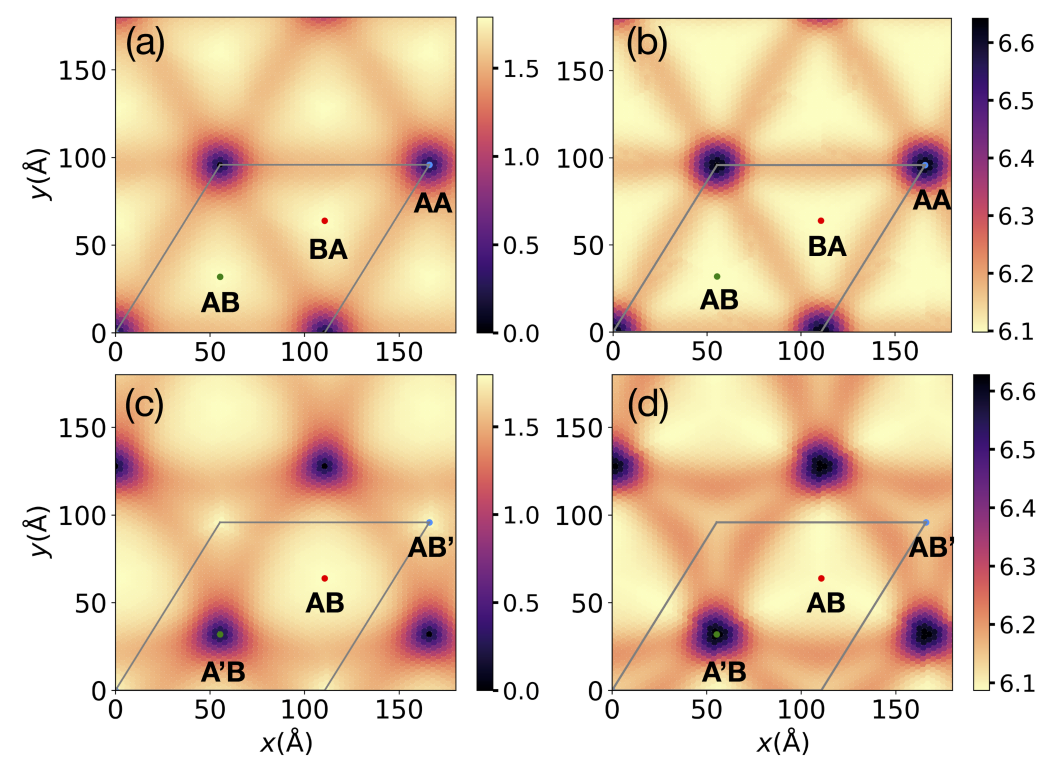

Figure 3: a and b (c and d) Order-parameter distribution and interlayer spacing distribution in $1.61^{\circ}\left(58.39^{\circ}\right)$ twisted bilayer $\mathrm{MoS}_{2}$, respectively. 


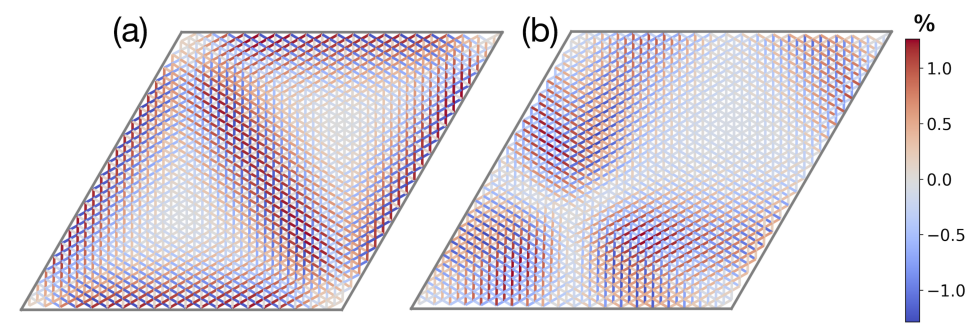

Figure 4: a and b Distribution of strain in the bottom layer of $1.61^{\circ}$ and $58.39^{\circ}$ ) twisted bilayer $\mathrm{MoS}_{2}$. Lines are drawn between every Mo atom and its 6 nearest neighbors. The color of the line represents the strain in that direction.

\section{Workflow}

In this section the sequential steps for operating the code are described. The code can be divided into three sections.

\subsection{Finding commensurate angle}

This part of the package is used to generate the commensurate twistangles and the corresponding lattice vectors using the method described in section 1. The code, get_ang.py, is used for this purpose and uses an input file: get_ang.inp.

1. Prepare input file, get_ang.inp. The lattice vectors for the top and bottom layer are provided by the user in this file. The basis atom positions are not necessary at this stage. The input file is also designed to contain an exhaustive range of constraints for the superlattice to be found. The user can fix the angle between the superlattice vectors, the type and amount of strain applied on the layer parameters, the tolerance threshold, $\Delta$. The user also provides a range of twist-angles to be searched through this input file. On running the code, a plot is generated with the lattice points and the superlattice vectors. Vectors of the same color show super-cells of the same area. An output file is generated for every twist-angle in the range provided by the user and contains all the solutions found by solving Eqn. 5 .

2. If too many solutions are found, the parameters can be changed to reduce the number of vectors and plot them for confirmation. Usually, it involves reducing ranges of $n_{1}, n_{2}, m_{1}, m_{2}$ to look for smaller superlattices or adjusting the strain percent and mismatch allowed. If no superlattice vectors are found, changes to the input are suggested. 
3. Pick the appropriate super-cell vectors from the output and prepare the second input file, twist.inp, to generate the atom positions.

\subsection{Atoms in superlattice}

This part of the package, twister.py, generates the atom coordinates in the moiré superlattice. The input files needed are twist.inp and basis atom positions of the unit-cell for top and bottom layer. The code also verifies that the atoms lying on the boundary of the supercell are not missed or counted twice. The code verifies that the number of atoms computed matches the expected value from area ratios. The expected number of atoms in top layer is given by: $n_{t o p}=n_{b} * \mathrm{Ar}_{s l} / \mathrm{Ar}_{u c}$, where $n_{b}$ is the number of basis atoms in top layer, $\mathrm{Ar}_{s l}$ is the area of the superlattice and $\mathrm{Ar}_{u c}$ is the area of the unit-cell. The output file, superlattice.dat, contains the superlattice vectors and the atom coordinates.

A flowchart summary of all the above steps can be found in Figure 5 .

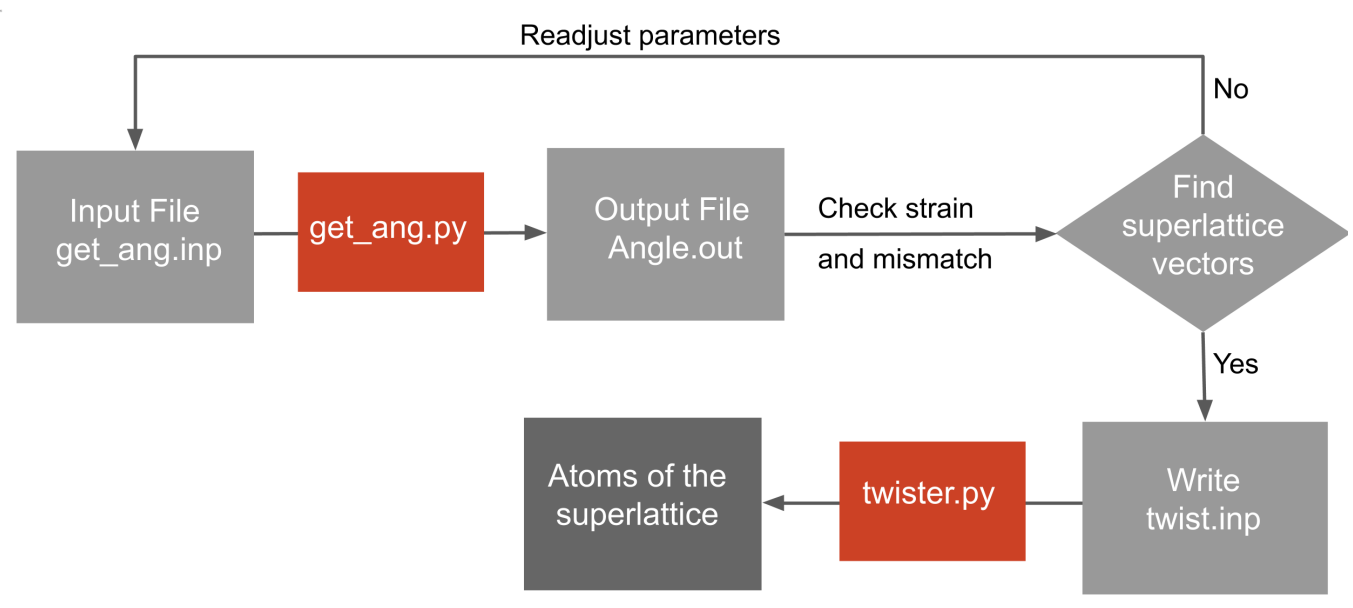

Figure 5: The program flow for generating the supercell lattice and number of atoms for given material and twist angle. 
(a)

(b)
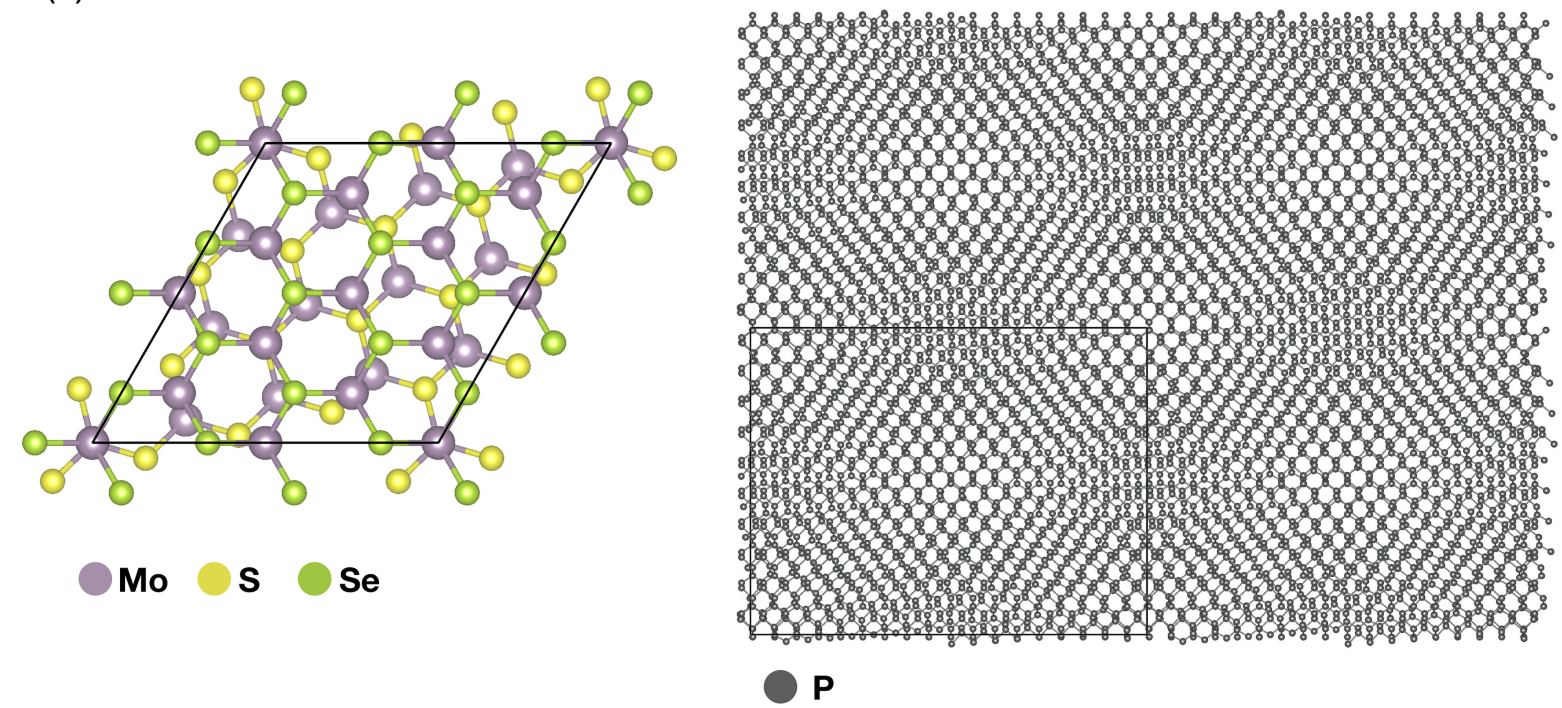

Figure 6: Superlattices of a) $16.1^{\circ}$ twisted $\mathrm{MoS}_{2} / \mathrm{MoSe}_{2}$ and b) $4.265^{\circ}$ twisted bilayer black phosphorous.

\section{Twister code framework}

\subsection{Input file}

We provide an example input file, get_ang.inp for the code get_ang.py described in the previous section to showcase the various options available to the user.

range_nm:

$-2021$

celldm_a:

3.1643 .16425 .0

a1:

$0.5 \quad 0.8660254 \quad 0.0$

a2:

$\begin{array}{lllll}-0.5 & 0.8660254 & 0.0\end{array}$

a3: 


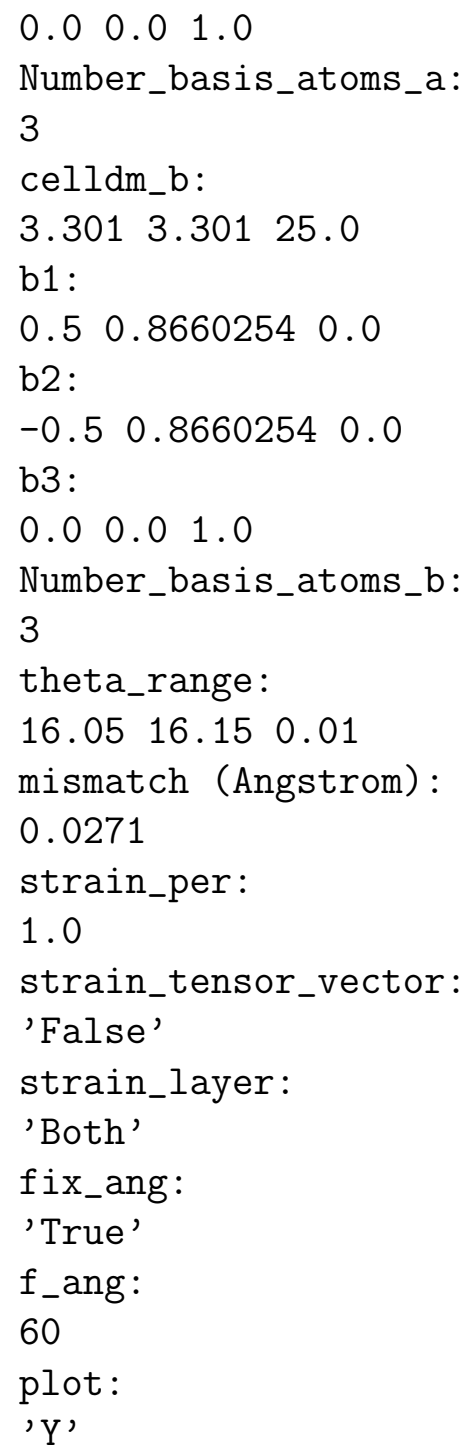

The unit of length is Angstrom $(\AA)$. Lattice parameters, unit-cell vectors and basis atoms for the top layer are provided in the line following the key-word: celldm_a,(a1,a2,a3) and Number_basis_atoms_a respectively and similarly for the bottom layer. Lower bound, upper bound and step size for the twist-angle range is given by the string below theta_range in the same order. Threshold for mismatch between coincident vectors of top and bottom layer can be specified in the string below mismatch (Angstroms). The user can apply a strain tensor by specifying 'True' below strain_tensor_vector 
otherwise, if specified 'False', only the lattice parameters (of the layer specified under strain_layer) will be strained. Threshold for percentage strain allowed to improve mismatch is given below strain_per. When entry under strain_tensor_vector is 'True', the deformation tensor method described above will be used. Fixing angle between superlattice vectors and specifying the angle between superlattice vectors are allowed by fix_ang and f_ang respectively. The user can plot the resultant superlattice vectors for each angle by specifying ' $Y$ ' below plot. Exact format of input can be found in the README files provided with each example included with the package.

\section{Parallelization and optimization}

The primary function for finding superlattice vectors in get_ang.py is the funtion $\operatorname{clt}()$ and scales as $\mathrm{O}\left(\mathrm{N}^{4}\right)$. We therefore optimise and parallelize this part of the code. Consider the unit-cell lattice vectors of bottom layer to be $\mathbf{a}_{1}, \mathbf{a}_{2}, \mathbf{a}_{3}$ and of top layer to be $\mathbf{b}_{1}, \mathbf{b}_{2}, \mathbf{b}_{3}$. Let superlattice in bottom layer be given as $\mathbf{v}_{1}=n_{1} \mathbf{a}_{1}+n_{2} \mathbf{a}_{2}$, and top layer as $\mathbf{v}_{2}=m_{1} \mathbf{b}_{1}+m_{2} \mathbf{b}_{2}$. The function clt () utilises user defined unit-cell lattice vectors and range of $n_{1}, n_{2}, m_{1}, m_{2}$ to checks all possible combinations of superlattice vectors for solutions. To parallelize computing the permutations, the range is scattered across the cores via MPI, using package mpi4py. Each core gets $N_{n m} / N_{c}$ numbers from the range, where $N_{n m}$ is the length of range of $n_{1}, n_{2}, m_{1}, m_{2}$ and $N_{n m}$ is the user-defined number of cores. For each element in its assigned data, the core generates a four-columned NumPy array, each column corresponding to $n_{1}, n_{2}, m_{1}, m_{2}$. The column for $m_{2}$ is uni-valued and only contains corresponding element in the data assigned to the core. The rows for $n_{1}, n_{2}, m_{1}$ contain all possible ordered triplets from the range of $n_{1}, n_{2}, m_{1}, m_{2}$ (with repeats allowed). These triplets were made using the product() iterator from the Itertools module of Python. A schematic for the the parallelized permutation calculations can be found in Figure 7 .

Using NumPy operations, the columns for $n_{1}, n_{2}$ are vector multiplied with $\mathbf{a}_{1}, \mathbf{a}_{2}$ and columns for $m_{1}, m_{2}$ are vector multiplied with rotated top layer vectors, $\mathbf{b}_{1}^{r}, \mathbf{b}_{2}^{r}$. The resulting NumPy arrays, $\mathbf{v}_{1}$ and $\mathbf{v}_{2}^{r}$, are used to calculate mismatch between them and the arrays are filtered according to the user-defined threshold (Eqn. 5). NumPy arrays for $\mathbf{v}_{1}, \mathbf{v}_{2}^{r},\left(n_{1}, n_{2}\right)$, $\left(m_{1}, m_{2}\right)$ and mismatch between the pairs, are sent by different cores and received by the core with $r a n k=0$. All possible pairs of superlattice vectors are then made using combinations() iterator from the Itertools module is 


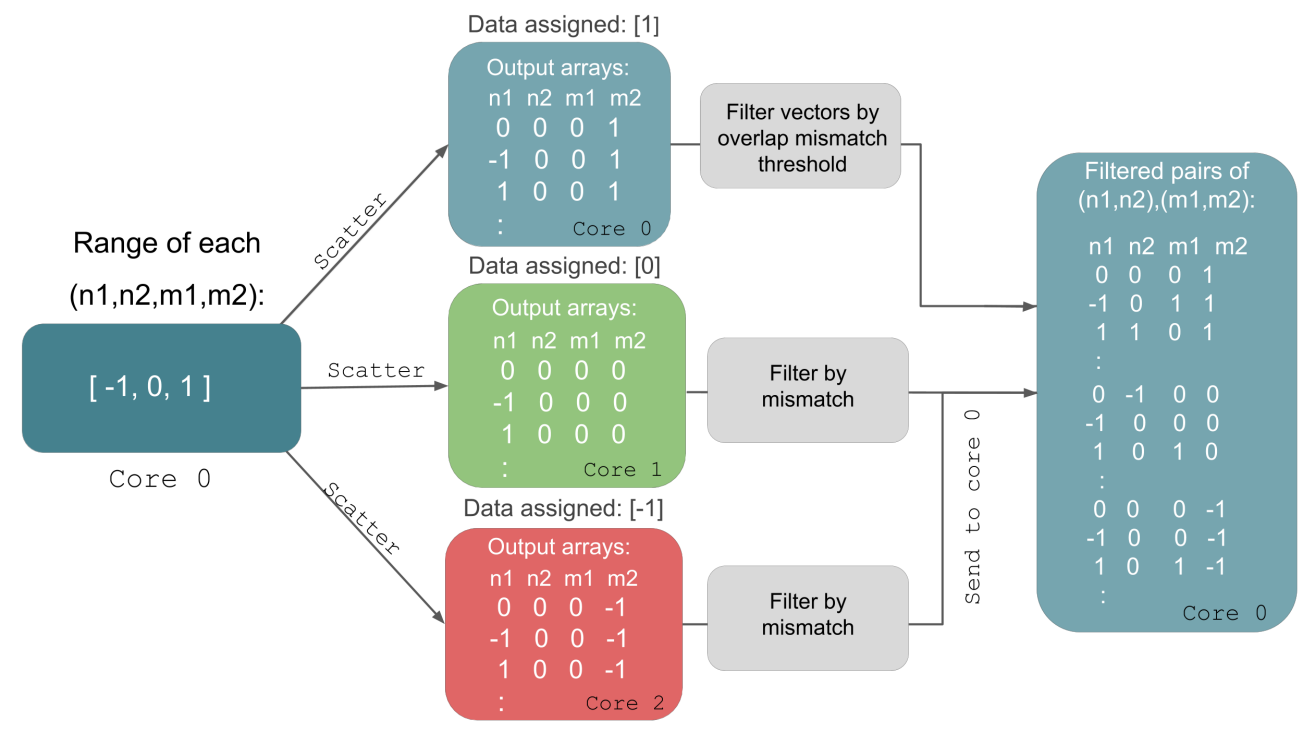

Figure 7: Schematic of the parallelization used in Twister to find commensurate twist angles.

used to make all possible pairs of superlattice vectors. They are filtered for non-parallel pairs. No parallelization is applied in the remaining steps. NumPy operations and arrays are used for further filtering depending on angle between superlattice vectors, strain in lattice parameters, and area of supercell.

\section{Conclusion}

We present the Twister package to construct commensurate moiré superlattice for any combination of 2D material. The package also streamlines atomic relaxation of the moiré superlattices by interfacing with classical forcefield calculations implemented in the LAMMPS package. We demonstrate the construction of the superlattices for five test systems, twisted bilayer $\mathrm{MoS}_{2}, \mathrm{MoS}_{2} / \mathrm{MoSe}_{2}$, graphene/hBN, hBN/MoSe 2 and twisted bilayer phosphorene. We also use the package to find commensurate twist-angles for the phosphorene bilayer and $\mathrm{MoS}_{2} / \mathrm{MoSe}_{2}$ heterobilayer. The package is written in Python and uses MPI parallelization for the computationally demanding steps. 


\section{Acknowledgments}

The authors thank the Supercomputer Education and Research Centre (SERC) at IISc for providing the computational facilities.

\section{References}

\section{References}

[1] Y. Cao, D. Rodan-Legrain, O. Rubies-Bigorda, J. M. Park, K. Watanabe, T. Taniguchi, P. Jarillo-Herrero, Tunable correlated states and spinpolarized phases in twisted bilayer-bilayer graphene, Nature 583 (7815) (2020) 215-220.

[2] Y. Choi, J. Kemmer, Y. Peng, A. Thomson, H. Arora, R. Polski, Y. Zhang, H. Ren, J. Alicea, G. Refael, F. von Oppen, K. Watanabe, T. Taniguchi, S. Nadj-Perge, Electronic correlations in twisted bilayer graphene near the magic angle, Nature Physics 15 (11) (2019) 11741180 .

[3] F. Haddadi, Q. Wu, A. J. Kruchkov, O. V. Yazyev, Moiré flat bands in twisted double bilayer graphene, Nano Letters 20 (4) (2020) 2410-2415, pMID: 32097013. doi:10.1021/acs.nanolett.9b05117.

[4] N. R. Chebrolu, B. L. Chittari, J. Jung, Flat bands in twisted double bilayer graphene, Phys. Rev. B 99 (2019) 235417. doi:10.1103/ PhysRevB.99.235417.

[5] L. Xian, D. M. Kennes, N. Tancogne-Dejean, M. Altarelli, A. Rubio, Multiflat bands and strong correlations in twisted bilayer boron nitride: Doping-induced correlated insulator and superconductor, Nano Letters 19 (8) (2019) 4934-4940, pMID: 31260633. doi:10.1021/acs. nanolett.9b00986.

[6] D. M. Kennes, L. Xian, M. Claassen, A. Rubio, One-dimensional flat bands in twisted bilayer germanium selenide, Nature communications 11 (1) (2020) 1-8.

[7] E. C. Regan, D. Wang, C. Jin, M. I. B. Utama, B. Gao, X. Wei, S. Zhao, W. Zhao, Z. Zhang, K. Yumigeta, et al., Mott and generalized wigner crystal states in wse 2/ws 2 moiré superlattices, Nature 579 (7799) (2020) 359-363. 
[8] B. Lian, Z. Wang, B. A. Bernevig, Twisted bilayer graphene: A phonondriven superconductor, Phys. Rev. Lett. 122 (2019) 257002. doi:10. 1103/PhysRevLett.122.257002.

[9] L. Wang, E.-M. Shih, A. Ghiotto, L. Xian, D. A. Rhodes, C. Tan, M. Claassen, D. M. Kennes, Y. Bai, B. Kim, K. Watanabe, T. Taniguchi, X. Zhu, J. Hone, A. Rubio, A. N. Pasupathy, C. R. Dean, Correlated electronic phases in twisted bilayer transition metal dichalcogenides, Nature Materials 19 (8) (2020) 861-866.

[10] F. Wu, T. Lovorn, E. Tutuc, I. Martin, A. H. MacDonald, Topological insulators in twisted transition metal dichalcogenide homobilayers, Phys. Rev. Lett. 122 (2019) 086402.

[11] Y. Cao, V. Fatemi, S. Fang, K. Watanabe, T. Taniguchi, E. Kaxiras, P. Jarillo-Herrero, Unconventional superconductivity in magic-angle graphene superlattices, Nature 556 (7699) (2018) 43-50.

[12] Y. Cao, V. Fatemi, A. Demir, S. Fang, S. L. Tomarken, J. Y. Luo, J. D. Sanchez-Yamagishi, K. Watanabe, T. Taniguchi, E. Kaxiras, R. C. Ashoori, P. Jarillo-Herrero, Correlated insulator behaviour at half-filling in magic-angle graphene superlattices, Nature 556 (7699) (2018) 80-84.

[13] S. Carr, D. Massatt, S. B. Torrisi, P. Cazeaux, M. Luskin, E. Kaxiras, Relaxation and domain formation in incommensurate two-dimensional heterostructures, Phys. Rev. B 98 (2018) 224102.

[14] F. Gargiulo, O. V. Yazyev, Structural and electronic transformation in low-angle twisted bilayer graphene, 2D Materials 5 (1) (2018) 015019.

[15] M. M. van Wijk, A. Schuring, M. I. Katsnelson, A. Fasolino, Relaxation of moiré patterns for slightly misaligned identical lattices: graphene on graphite, 2D Materials 2 (3) (2015) 034010.

[16] I. Maity, P. K. Maiti, H. R. Krishnamurthy, M. Jain, Reconstruction of moiré lattices in twisted transition metal dichalcogenide bilayers (2019). arXiv: 1912.08702.

[17] M. H. Naik, M. Jain, Ultraflatbands and shear solitons in moiré patterns of twisted bilayer transition metal dichalcogenides, Phys. Rev. Lett. 121 (2018) 266401. 
[18] V. V. Enaldiev, V. Zólyomi, C. Yelgel, S. J. Magorrian, V. I. Fal'ko, Stacking domains and dislocation networks in marginally twisted bilayers of transition metal dichalcogenides, Phys. Rev. Lett. 124 (2020) 206101. doi:10.1103/PhysRevLett.124.206101.

URL https://link.aps.org/doi/10.1103/PhysRevLett.124. 206101

[19] X. Liang, Z. A. H. Goodwin, V. Vitale, F. Corsetti, A. A. Mostofi, J. Lischner, Effect of bilayer stacking on the atomic and electronic structure of twisted double bilayer graphene (2020). arXiv:2008.05269.

[20] F. J. Culchac, R. R. Del Grande, R. B. Capaz, L. Chico, E. S. Morell, Flat bands and gaps in twisted double bilayer graphene, Nanoscale 12 (2020) 5014-5020.

[21] P. Lucignano, D. Alfè, V. Cataudella, D. Ninno, G. Cantele, Crucial role of atomic corrugation on the flat bands and energy gaps of twisted bilayer graphene at the magic angle $\theta \sim 1.08^{\circ}$, Phys. Rev. B 99 (2019) 195419.

[22] S. K. Jain, V. Juričić, G. T. Barkema, Structure of twisted and buckled bilayer graphene, 2D Materials 4 (1) (2016) 015018.

[23] N. Leconte, S. Javvaji, J. An, J. Jung, Relaxation Effects in Twisted Bilayer Graphene: a Multi-Scale Approach, arXiv e-prints (2019) arXiv:1910.12805larXiv:1910.12805.

[24] N. N. T. Nam, M. Koshino, Lattice relaxation and energy band modulation in twisted bilayer graphene, Phys. Rev. B 96 (2017) 075311.

[25] A. Lopez-Bezanilla, J. L. Lado, Electrical band flattening, valley flux, and superconductivity in twisted trilayer graphene, arXiv e-prints (2020) arXiv:2005.02169larXiv:2005.02169.

[26] J. S. Alden, A. W. Tsen, P. Y. Huang, R. Hovden, L. Brown, J. Park, D. A. Muller, P. L. McEuen, Strain solitons and topological defects in bilayer graphene, Proceedings of the National Academy of Sciences 110 (28) (2013) 11256-11260. 
[27] M. R. Rosenberger, H.-J. Chuang, M. Phillips, V. P. Oleshko, K. M. McCreary, S. V. Sivaram, C. S. Hellberg, B. T. Jonker, Twist angledependent atomic reconstruction and moiré patterns in transition metal dichalcogenide heterostructures, ACS Nano 14 (4) (2020) 4550-4558, pMID: 32167748. doi:10.1021/acsnano.0c00088.

[28] H. Yoo, R. Engelke, S. Carr, S. Fang, K. Zhang, P. Cazeaux, S. H. Sung, R. Hovden, A. W. Tsen, T. Taniguchi, K. Watanabe, G.-C. Yi, M. Kim, M. Luskin, E. B. Tadmor, E. Kaxiras, P. Kim, Atomic and electronic reconstruction at the van der waals interface in twisted bilayer graphene, Nature Materials 18 (5) (2019) 448-453.

[29] H. Li, S. Li, M. H. Naik, J. Xie, X. Li, J. Wang, E. Regan, D. Wang, W. Zhao, S. Zhao, S. Kahn, K. Yumigeta, M. Blei, T. Taniguchi, K. Watanabe, S. Tongay, A. Zettl, S. G. Louie, F. Wang, M. F. Crommie, Imaging moiréflat bands in three-dimensional reconstructed wse2/ws2 superlattices, Nature Materials.

[30] A. Weston, Y. Zou, V. Enaldiev, A. Summerfield, N. Clark, V. Zólyomi, A. Graham, C. Yelgel, S. Magorrian, M. Zhou, J. Zultak, D. Hopkinson, A. Barinov, T. H. Bointon, A. Kretinin, N. R. Wilson, P. H. Beton, V. I. Fal'ko, S. J. Haigh, R. Gorbachev, Atomic reconstruction in twisted bilayers of transition metal dichalcogenides, Nature Nanotechnology 15 (7) (2020) 592-597.

[31] C. R. Woods, L. Britnell, A. Eckmann, R. S. Ma, J. C. Lu, H. M. Guo, X. Lin, G. L. Yu, Y. Cao, R. V. Gorbachev, A. V. Kretinin, J. Park, L. A. Ponomarenko, M. I. Katsnelson, Y. N. Gornostyrev, K. Watanabe, T. Taniguchi, C. Casiraghi, H.-J. Gao, A. K. Geim, K. S. Novoselov, Commensurate-incommensurate transition in graphene on hexagonal boron nitride, Nature Physics 10 (6) (2014) 451-456.

[32] L. J. McGilly, A. Kerelsky, N. R. Finney, K. Shapovalov, E.-M. Shih, A. Ghiotto, Y. Zeng, S. L. Moore, W. Wu, Y. Bai, K. Watanabe, T. Taniguchi, M. Stengel, L. Zhou, J. Hone, X. Zhu, D. N. Basov, C. Dean, C. E. Dreyer, A. N. Pasupathy, Visualization of moirésuperlattices, Nature Nanotechnology 15 (7) (2020) 580-584.

[33] P. Kang, W.-T. Zhang, V. Michaud-Rioux, X.-H. Kong, C. Hu, G.H. Yu, H. Guo, Moiré impurities in twisted bilayer black phosphorus: 
Effects on the carrier mobility, Phys. Rev. B 96 (2017) 195406. doi: 10.1103/PhysRevB.96.195406.

[34] S. Carr, S. Fang, Z. Zhu, E. Kaxiras, Exact continuum model for lowenergy electronic states of twisted bilayer graphene, Phys. Rev. Research 1 (2019) 013001.

[35] X.-J. Zhao, Y. Yang, D.-B. Zhang, S.-H. Wei, Formation of bloch flat bands in polar twisted bilayers without magic angles, Phys. Rev. Lett. 124 (2020) 086401. doi:10.1103/PhysRevLett.124.086401.

[36] M. H. Naik, S. Kundu, I. Maity, M. Jain, Origin and evolution of ultraflat bands in twisted bilayer transition metal dichalcogenides: Realization of triangular quantum dots, Phys. Rev. B 102 (2020) 075413. doi: 10.1103/PhysRevB.102.075413. URL https://link.aps .org/doi/10.1103/PhysRevB .102.075413

[37] M. I. B. Utama, R. J. Koch, K. Lee, N. Leconte, H. Li, S. Zhao, L. Jiang, J. Zhu, K. Watanabe, T. Taniguchi, P. D. Ashby, A. Weber-Bargioni, A. Zettl, C. Jozwiak, J. Jung, E. Rotenberg, A. Bostwick, F. Wang, Visualization of the flat electronic band in twisted bilayer graphene near the magic angle twist, Nature Physics.

[38] M. Angeli, A. H. MacDonald, $\gamma$-valley transition-metal-dichalcogenide moirè bands (2020). arXiv:2008.01735.

[39] L. Xian, M. Claassen, D. Kiese, M. M. Scherer, S. Trebst, D. M. Kennes, A. Rubio, Realization of nearly dispersionless bands with strong orbital anisotropy from destructive interference in twisted bilayer mos2 (2020). arXiv:2004.02964.

[40] M. Koshino, Y.-W. Son, Moiré phonons in twisted bilayer graphene, Phys. Rev. B 100 (2019) 075416. doi:10.1103/PhysRevB.100.075416.

[41] I. Maity, M. H. Naik, P. K. Maiti, H. R. Krishnamurthy, M. Jain, Phonons in twisted transition-metal dichalcogenide bilayers: Ultrasoft phasons and a transition from a superlubric to a pinned phase, Phys. Rev. Research 2 (2020) 013335. doi:10.1103/PhysRevResearch.2. 013335 . 
[42] R. Debnath, I. Maity, R. Biswas, V. Raghunathan, M. Jain, A. Ghosh, Evolution of high-frequency raman modes and their doping dependence in twisted bilayer mos2, Nanoscale (2020)-doi:10.1039/C9NR09897F.

[43] W. Kohn, L. J. Sham, Self-consistent equations including exchange and correlation effects, Phys. Rev. 140 (1965) A1133-A1138.

[44] K. Uchida, S. Furuya, J.-I. Iwata, A. Oshiyama, Atomic corrugation and electron localization due to moiré patterns in twisted bilayer graphenes, Phys. Rev. B 90 (2014) 155451.

[45] J. M. B. Lopes dos Santos, N. M. R. Peres, A. H. Castro Neto, Graphene bilayer with a twist: Electronic structure, Phys. Rev. Lett. 99 (2007) 256802. doi:10.1103/PhysRevLett.99.256802.

[46] D. S. Koda, F. Bechstedt, M. Marques, L. K. Teles, Coincidence lattices of $2 \mathrm{~d}$ crystals: Heterostructure predictions and applications, The Journal of Physical Chemistry C 120 (20) (2016) 10895-10908. doi:10.1021/acs.jpcc.6b01496.

[47] C. Zhang, C.-P. Chuu, X. Ren, M.-Y. Li, L.-J. Li, C. Jin, M.-Y. Chou, C.-K. Shih, Interlayer couplings, moiré patterns, and $2 \mathrm{~d}$ electronic superlattices in mos2/wse2 hetero-bilayers, Science Advances 3 (1).

[48] D. Waters, Y. Nie, F. Lüpke, Y. Pan, S. Fölsch, Y.-C. Lin, B. Jariwala, K. Zhang, C. Wang, H. Lv, K. Cho, D. Xiao, J. A. Robinson, R. M. Feenstra, Flat bands and mechanical deformation effects in the moiré superlattice of mos2-wse2 heterobilayers, ACS Nano 14 (6) (2020) 75647573, pMID: 32496750.

[49] S. Dai, Y. Xiang, D. J. Srolovitz, Twisted bilayer graphene: Moiré with a twist, Nano Letters 16 (9) (2016) 5923-5927.

[50] B. Butz, C. Dolle, F. Niekiel, K. Weber, D. Waldmann, H. B. Weber, B. Meyer, E. Spiecker, Dislocations in bilayer graphene, Nature 505 (7484) (2014) 533-537.

[51] F. Wu, T. Lovorn, E. Tutuc, A. H. MacDonald, Hubbard model physics in transition metal dichalcogenide moiré bands, Phys. Rev. Lett. 121 (2018) 026402. doi:10.1103/PhysRevLett.121.026402. 
URL

https://link.aps.org/doi/10.1103/PhysRevLett.121. 026402

[52] H. Pan, F. Wu, S. Das Sarma, Band topology, hubbard model, heisenberg model, and dzyaloshinskii-moriya interaction in twisted bilayer wse $_{2}$, Phys. Rev. Research 2 (2020) 033087.

[53] Y. Zhang, N. F. Q. Yuan, L. Fu, Moiré quantum chemistry: charge transfer in transition metal dichalcogenide superlattices, arXiv e-prints (2019) arXiv:1910.14061|arXiv:1910.14061.

[54] Z. Bi, N. F. Q. Yuan, L. Fu, Designing flat bands by strain, Phys. Rev. B 100 (2019) 035448.

[55] F. Wu, T. Lovorn, A. H. MacDonald, Topological exciton bands in moiré heterojunctions, Phys. Rev. Lett. 118 (2017) 147401.

[56] F. H. Stillinger, T. A. Weber, Computer simulation of local order in condensed phases of silicon, Phys. Rev. B 31 (1985) 5262-5271.

[57] A. N. Kolmogorov, V. H. Crespi, Registry-dependent interlayer potential for graphitic systems, Phys. Rev. B 71 (2005) 235415.

[58] J.-W. Jiang, Y.-P. Zhou, Parameterization of stillinger-weber potential for two- dimensional atomic crystals, in: J.-W. Jiang, Y.-P. Zhou (Eds.), Handbook of Stillinger-Weber Potential Parameters for TwoDimensional Atomic Crystals, IntechOpen, Rijeka, 2017, Ch. 1.

[59] M. H. Naik, I. Maity, P. K. Maiti, M. Jain, Kolmogorov-crespi potential for multilayer transition-metal dichalcogenides: Capturing structural transformations in moiré superlattices, The Journal of Physical Chemistry C 123 (15) (2019) 9770-9778. doi:10.1021/acs.jpcc.8b10392.

[60] I. Leven, T. Maaravi, I. Azuri, L. Kronik, O. Hod, Interlayer potential for graphene/h-bn heterostructures, Journal of Chemical Theory and Computation 12 (6) (2016) 2896-2905, pMID: 27168429.

[61] T. Maaravi, I. Leven, I. Azuri, L. Kronik, O. Hod, Interlayer potential for homogeneous graphene and hexagonal boron nitride systems: Reparametrization for many-body dispersion effects, The Journal of Physical Chemistry C 121 (41) (2017) 22826-22835. 
[62] M. Wen, S. Carr, S. Fang, E. Kaxiras, E. B. Tadmor, Dihedral-anglecorrected registry-dependent interlayer potential for multilayer graphene structures, Phys. Rev. B 98 (2018) 235404.

[63] I. Leven, I. Azuri, L. Kronik, O. Hod, Inter-layer potential for hexagonal boron nitride, The Journal of Chemical Physics 140 (10) (2014) 104106.

[64] S. Plimpton, Fast parallel algorithms for short-range molecular dynamics, Journal of Computational Physics 117 (1) (1995) 1 - 19.

[65] R. A. Horn, C. R. Johnson, Matrix analysis, Cambridge university press, 2012 .

[66] A. Stukowski, Visualization and analysis of atomistic simulation data with OVITO-the Open Visualization Tool, MODELLING AND SIMULATION IN MATERIALS SCIENCE AND ENGINEERING 18 (1). doi: $\{10.1088 / 0965-0393 / 18 / 1 / 015012\}$. 